\title{
Spatial Relationship of Signatures of Interplanetary Coronal Mass Ejections
}

\author{
I. G. Richardson ${ }^{1}$, H. V. Cane ${ }^{1}$, S. T. Lepri ${ }^{2}$, T. H. Zurbuchen ${ }^{2}$, and J. T. Gosling ${ }^{3}$ \\ ${ }^{1}$ Laboratory for High Energy Astrophysics, NASA/Goddard Space Flight Center, Greenbelt, MD 20771, USA, \\ ${ }^{2}$ Dept. of Atmospheric, Oceanic and Space Sciences, University of Michigan, Ann Arbor, MI 48109, USA; \\ ${ }^{3}$ Los Alamos National Laboratory, Los Alamos, NM 87545, USA
}

\begin{abstract}
Interplanetary coronal mass ejections (ICMEs) are characterized by a number of signatures. In particular, we examine the relationship between Fe charge states and other signatures during ICMEs in solar cycle 23 . Though enhanced Fe charge states characterize many ICMEs, average charge states vary from event to event, are more likely to be enhanced in faster or flare-related ICMEs, and do not appear to depend on whether the ICME is a magnetic cloud.
\end{abstract}

\section{INTRODUCTION}

Interplanetary coronal mass ejections (ICMEs), the counterparts in the solar wind of coronal mass ejections (CMEs) at the Sun, are characterized by various signatures (e.g., 1, 2, and references therein). These include: abnormally low solar wind proton temperatures $\left(T_{p}\right)$, bi-directional suprathermal electron strahls (BDEs), low plasma $\beta$, helium abundance enhancements, low magnetic field variances, nonParker-spiral field directions and, in a subset of events, the enhanced, smoothly-rotating magnetic fields and low $\beta$ plasma defining "magnetic clouds". Energetic particle signatures include intensity depressions (cosmic ray Forbush decreases) and bi-directional flows at $\sim \mathrm{MeV}$ to cosmic ray energies (e.g., 3). Each ICME is unique to some extent and most do not exhibit all of the characteristic signatures. Thus, there is no obvious "necessary and sufficient" condition for the identification of an ICME. In addition, as has been well known for many years (e.g., 4), the boundaries of the various signatures may not be co-located. Hence, a number of signatures should be compared in order to arrive at a consensus. From considering these various ICME signatures, we have identified $\sim 200$ probable ICMEs in the near-Earth solar wind since beginning of the current solar cycle in 1996 until the end of 2001. Detailed observations of plasma composition anomalies in many of these ICMEs are available from the SWICS instrument on ACE. Recently, Lepri et al.
(5) have reported that intervals of enhanced iron charge states with durations $\geq 20$ hours observed in 1998-mid 2000 were associated with $\sim 50 \%$ of the ICMEs that we identified independently during this period. The high $\mathrm{Fe}$ charge states, which remain unchanged ("frozen-in") as the solar wind propagates through the heliosphere, are consistent with coronal temperatures often exceeding $\sim 2 \mathrm{MK}$ and rapid plasma expansion during CME initiation. The emphasis in this paper is on examining further the relationship between enhanced $\mathrm{Fe}$ charge states and ICMEs, including their relative boundaries. Since a significant fraction of ICMEs do not appear to be associated with Fe events as defined by (5), we will consider whether high $\mathrm{Fe}$ charge states are present in other ICMEs but for intervals $<20$ hours, whether they are absent in some ICMEs, and whether the characteristics of the ICME or the related solar event (such as the occurrence of a flare) may have any bearing on the $\mathrm{Fe}$ charge states inside an ICME.

\section{OBSERVATIONS}

The ICME of September 25-26, 1998 (Figure 1) is an example of a "classic", well-studied, event. It is not particularly typical however in that it exhibits a wide range of signatures that are essentially co-located. The main panel shows 5-minute averaged ACE observations of the magnetic field intensity and polar 


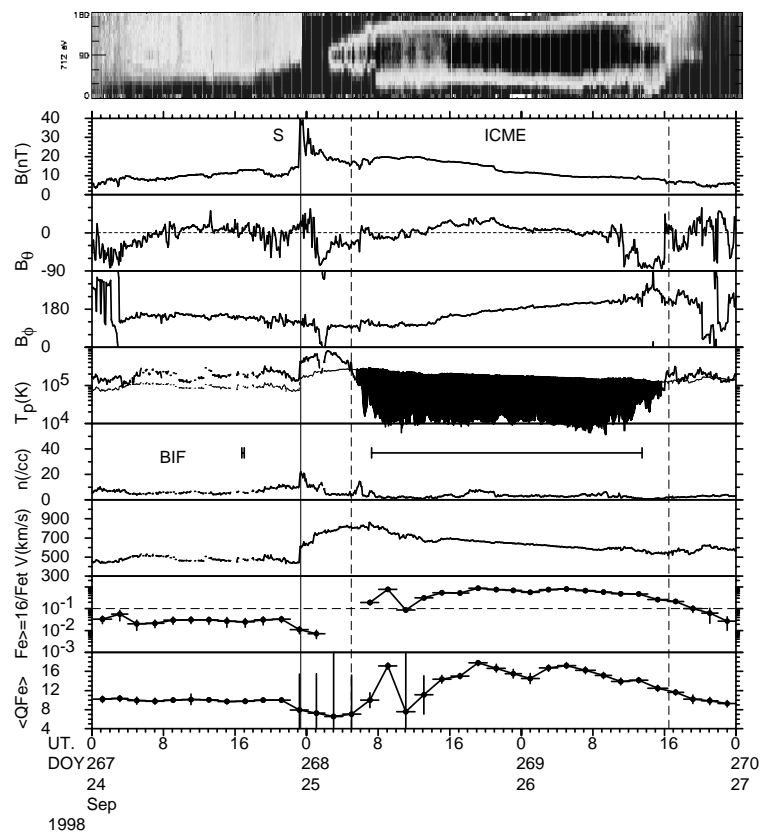

FIGURE 1. An ICME on 25-26 September, 1998 which drove a shock $(\mathrm{S})$ ahead of it (solid vertical line). $\mathrm{BIF}=$ bi-directional $\sim 1 \mathrm{MeV}$ ions observed by IMP 8 (6). The top panel shows pitch-angle distributions for $712 \mathrm{eV}$ solar wind electrons.

and azimuthal angles; the plasma proton temperature, density and speed; and two-hour averages of the ratio $\mathrm{Fe} \geq 16+/ \mathrm{Fe}_{\text {tot }}$, and the mean $\mathrm{Fe}$ charge state. In the $T_{p}$ panel, the second trace indicates the "expected temperature" for normally-expanding solar wind $\left(T_{e x}\right)$, which is correlated with the solar wind speed (2). Shading indicates when $T_{p}<0.5 T_{e x}$, frequently a signature of ICMEs (2). This fast ( $700 \mathrm{~km} / \mathrm{s})$ ICME generated a strong shock ahead of it and originated in association with an $\mathrm{M} 7 / 3 \mathrm{~B}$ flare at $\mathrm{N} 18^{\circ} \mathrm{E} 09^{\circ}$ on September 23. The vertical dashed lines are the ICME boundaries suggested by the low $T_{p}$ region. A discontinuity terminating an interval of southward magnetic field is evident at the trailing edge. The magnetic field in the ICME is relatively smooth and rotates slowly in azimuth. The enhanced $\mathrm{Fe}$ charge states coincide with this region, to within the 2-hour resolution of the measurements, and reach $\mathrm{Fe} \geq 16+/ \mathrm{Fe}_{\text {tot }} \sim 1$ and $\left\langle\mathrm{Q}_{\mathrm{Fe}}>\sim 16\right.$, indicating freeze-in temperatures $T_{F e} \sim 7 \mathrm{MK}(5)$. The top panel in Figure 1 shows pitch-angle distributions of $712 \mathrm{eV}$ solar wind electrons from the ACE/SWEPAM experiment. Bands at the top and bottom of the plot (clearer in the colored original) indicate electrons flowing in both directions along the magnetic field, i.e., a BDE. This starts $\sim 3$ hours ahead of the ICME leading edge based on $T_{p}$ and is consistent with an abrupt decrease in the $>60 \mathrm{MeV}$ cosmic ray intensity observed at IMP 8 (6), suggesting that entry into a closed magnetic structure, and the true

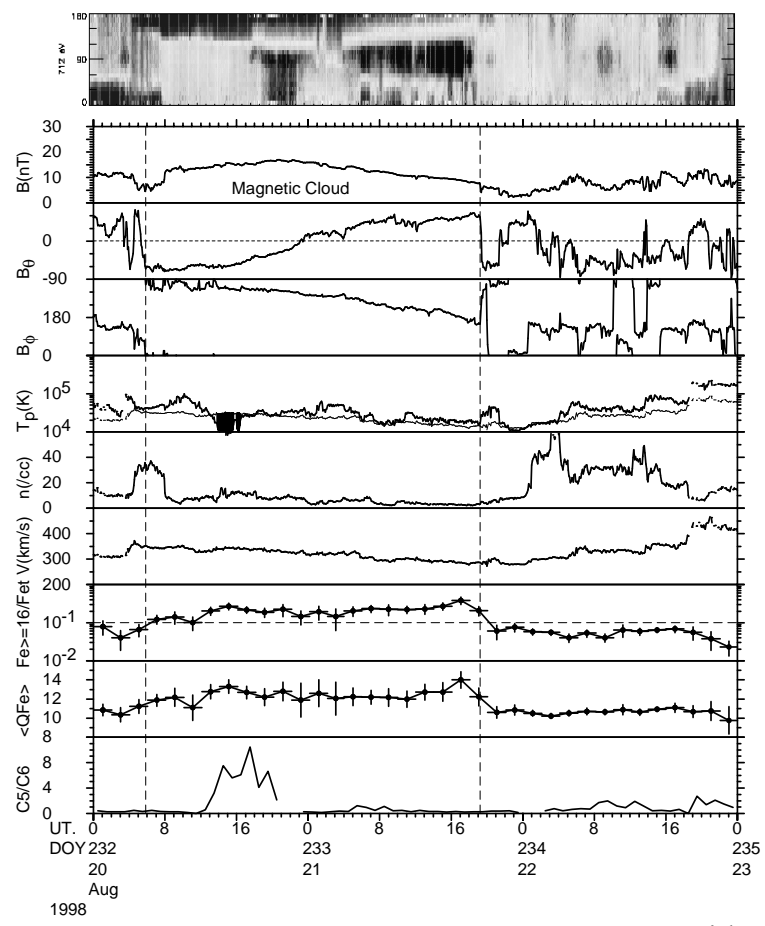

FIGURE 2. An ICME on 20-21 August, 1998 with a "magnetic cloud" configuration.

ICME leading edge, most likely occurred at this time. The interval of bi-directional $\sim 1 \mathrm{MeV}$ ion flows observed by IMP 8 in this ICME (6) is indicated by "BIF" in Figure 1.

An ICME on August 20-21, 1998 with the enhanced, smoothly rotating magnetic field characteristic of a magnetic cloud (MC) is shown in Figure 2. The MC interval has $F e \geq 16+/ F e_{\text {tot }} \geq 0.1$. However, this ratio $(\sim 0.2-0.3)$ and $\left\langle Q_{F \mathrm{e}}>\left(\sim 12-13 ; T_{F e}\right.\right.$ $\sim 1.5 \mathrm{MK})$ are significantly less than during the ICME in Figure 1. During most of the MC, $T_{p} \sim T_{e x}$. The brief period of low $T_{p}$ (shaded) clearly does not denote the true extent of the ICME. The $712 \mathrm{eV}$ electron distribution (top panel) indicates BDEs during a high density/depressed field region near the MC leading edge and intermittently during the trailing half of the MC. Elsewhere in the MC, the absence of BDEs suggests that magnetic fields were open (7). An interesting feature is the peak in the $\mathrm{C}^{5} / \mathrm{C}^{6}$ ratio associated with the low $T_{p}$ region. Similar features are seen in some other ICMEs (see Figure 3) and suggest that occasionally, regions of low $T_{p}$ at $1 \mathrm{AU}$ may reflect low coronal temperatures during ICME initiation, though this needs further investigation.

Another ICME (on August 26-27, 1998) is shown in Figure 3. This relatively fast ICME (associated with an $\mathrm{X} 1 / 3 \mathrm{~B}$ flare at $\mathrm{N} 35^{\circ} \mathrm{E} 09^{\circ}$ on August 24) also generated a shock ahead of it. The ICME interval is 


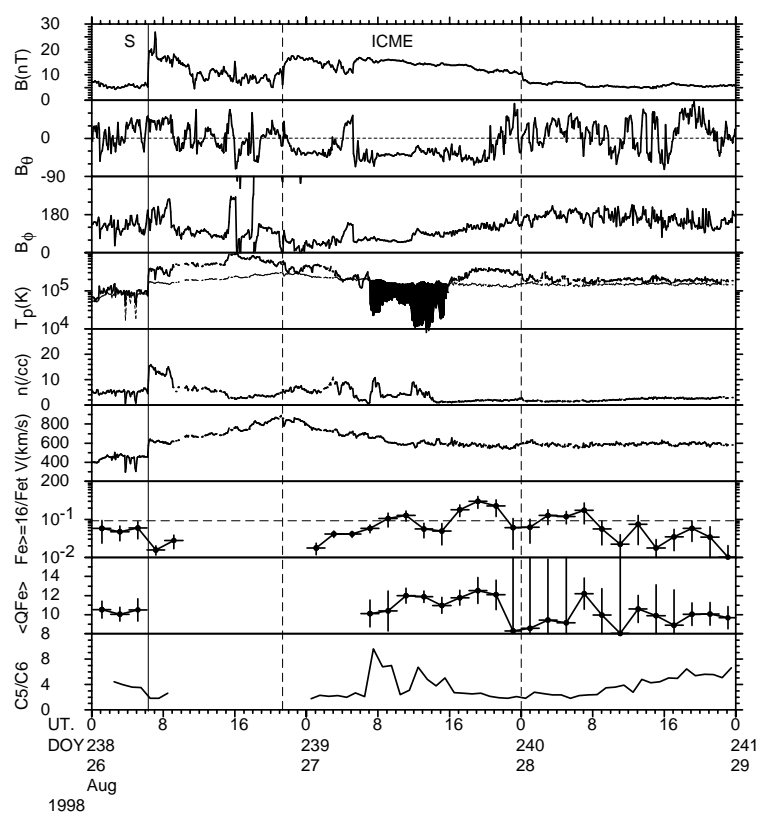

FIGURE 3. An ICME on 26-27 August, 1998 that generated a shock ahead of it. Note the lack of high Fe charge states compared to the ICME in Figure 1.

principally inferred from the enhanced, relatively lowvariance magnetic field commencing $\sim 15$ hours following the shock. Abnormally-low $T p$ only occupies the center third of this interval and, as in Figure 2 , is associated with enhanced $\mathrm{C}^{5} / \mathrm{C}^{6}$. Only a $\sim 6$ hour period inside the ICME trailing edge has $F e \geq 16+/ F e_{\text {tot }} \geq 0.1$, and average charge states within
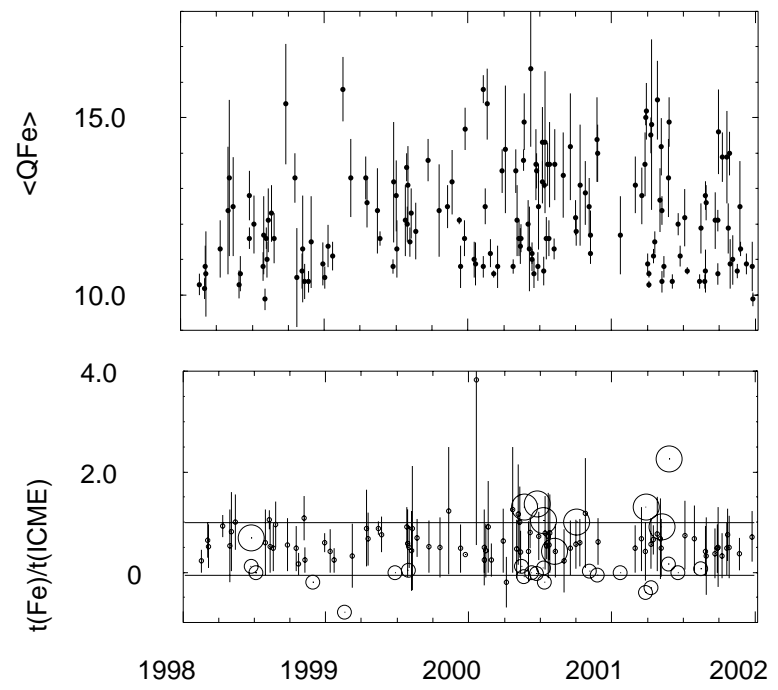

FIGURE 4. (a) Mean Fe charge states (average of 2$\mathrm{hr}$ averages) in 157 probable ICMEs in 1998-2001. (b) Times of intervals with $F e \geq 16+/ F_{\text {tot }} \geq 0.1$ relative to the ICME interval (0-1). Small (large) circles = start (end) time only available.
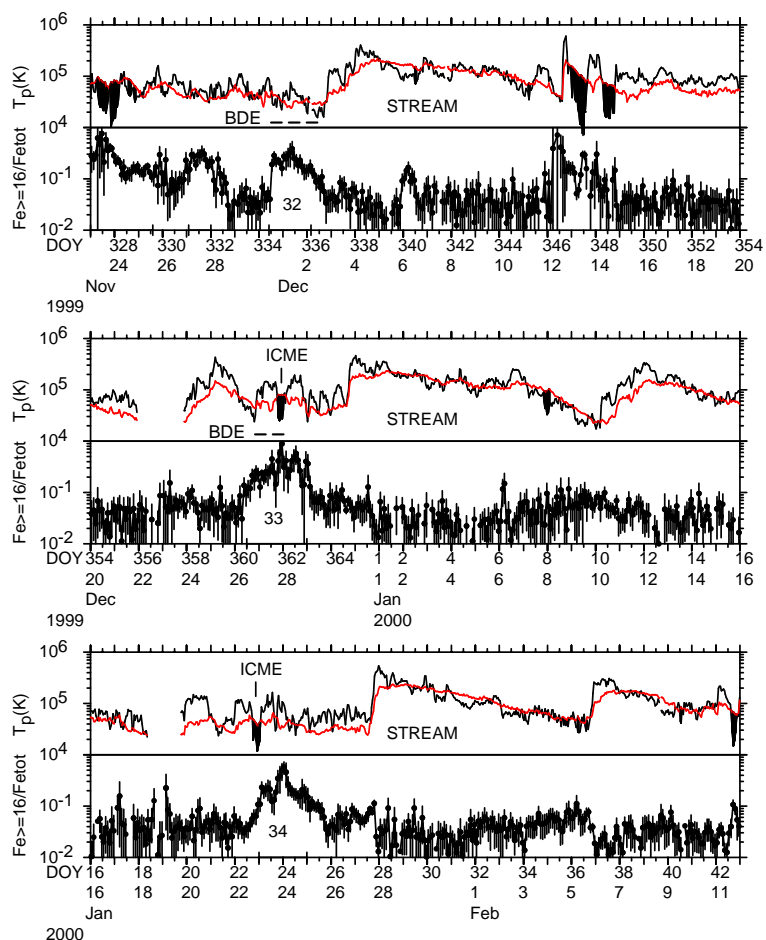

FIGURE 5. The middle panel shows an ICME embedded in an extended high Fe charge-state interval which appears to recur at the 27-day solar-rotation period (top and bottom panels). $T_{p}$ and $T_{\text {exp }}$ are shown in addition to $F e \geq 16+/ F e_{\text {tot }}$. Fe event numbers are from (4). BDEs are also indicated.

the ICME are 10-12 ( $\left.T_{F e} \leq 1.5 \mathrm{MK}\right)$. Though not shown here, a BDE was also present in this ICME.

The above observations suggest that Fe charge states vary from event to event, and enhanced charge states do not characterize every ICME. Figure 4(a) shows $<\mathrm{Q}_{\mathrm{Fe}}>$ (mean of 2-hr averages) during 157 probable ICMEs in 1998-2001. The all-event average is $12.2 \pm 1.5$, only slightly above the average for all solar wind during this period (11.1 \pm 1$)$. In 22 events $(14 \%),<\mathrm{Q}_{\mathrm{Fe}}>\geq 14\left(T_{\mathrm{Fe}}>2 \mathrm{MK}\right)$. A preliminary examination suggests that 10 of these high charge state ICMEs have associations with solar flares (C7-X6). A further 8 have probable associations, one was associated with a LASCO halo CME with no reported flare, and the associations are unclear for 2 events. Finally, one ICME was embedded in an extended high charge state event (December 26-28, 1999; middle panel of Figure 5) that appears to be part of a sequence of such events, nos. 28, and 32-34 of (5), that recur at approximately the solar rotation period and precede a corotating high-speed stream. Events 32-34 are shown in Figure 5. Event 32 was coincident with an extended BDE. A BDE was also present during the first part of Event 33, extending to the end of the low $T_{p}$ signature. 
To examine the spatial relationship between ICME boundaries (defined by a consensus of signatures) and enhanced Fe charge states, Figure 4(b) summarizes the locations of intervals with $\mathrm{Fe} \geq 16+/ F e_{\text {tot }} \geq 0.1$ relative to the ICME boundaries, normalized to the ICME duration (i.e., 0 corresponds to the ICME leading edge, and 1 to the trailing edge). The enhanced Fe charge states generally commence close to or inside the leading edge and finish inside the trailing edge. They do not favor specific locations in the ICME (e.g, inside the leading or trailing edge, or center). They tend to extend some distance beyond the ICME trailing edge more frequently than they do at the leading edge. This may be because it is generally more difficult to locate ICME trailing edges, often the Fe charge state ratio declines gradually to the $\sim 0.1$ threshold level, and the statistical accuracy of the $\mathrm{Fe}$ data will be reduced in the lower density plasma that is often present in ICME trailing edges. Nonetheless, an interesting possibility, which requires further study, is that a region of high $\mathrm{Fe}$ charge states follows some ICMEs. In a few cases, abrupt changes in $\mathrm{Fe}$ charge states occur within the ICME, suggestive of the presence of substructures, possibly multiple ICMEs. The most notable differences between the ICME and high Fe charge state intervals are for those ICMEs embedded in "recurrent" events as discussed above.

We have also examined whether there is any relationship between the mean $\mathrm{Fe}$ charge state and other ICME parameters. Around $30 \%$ of ICMEs have $<\mathrm{Q}_{\mathrm{Fe}}>\geq 13$, irrespective of whether they are "reported" magnetic clouds, have some evidence of a magnetic field rotation but have not been reported as magnetic clouds, or have no cloud-like magnetic signature. Thus, enhanced Fe charge states do not appear to depend on the presence or absence of a magnetic cloud structure. There is some evidence of a speed-dependence. Only $21 \%$ of ICMEs in our sample with in-situ speeds $<500 \mathrm{~km} / \mathrm{s}$ have $<\mathrm{Q}_{\mathrm{Fe}}>\geq 13$, compared with $56 \%$ of those with speeds $>500 \mathrm{~km} / \mathrm{s}$. There is also evidence of a solar-activity dependence. In $1998,10 \%$ of the ICMEs have $<\mathrm{Q}_{\mathrm{Fe}}>\geq 13$ compared with $32 \%$ in $1999,43 \%$ in 2000 (year of sunspot maximum), and $29 \%$ in 2001.

\section{SUMMARY AND DISCUSSION}

The finding (5) that only $\sim 50 \%$ of the ICMEs identified independently using other signatures had high $\mathrm{Fe}$ charge states (as defined by (5)) arises because such charge states are not present in all ICMEs, or, if present, are detected for less than the 20 hour criterion required by (5). The charge-states (and other observed ICME characteristics) will also be influenced by the spacecraft trajectory relative to the ICME. In general, high Fe charge states lie within the ICME, though they occasionally continue beyond the ICME trailing edge. ICMEs with high $\mathrm{Fe}$ charge states appear to be predominantly related to solar flares, although the event in Figure 3 indicates that not all flare-related events have high charge states. Faster ICMEs are also more likely to be associated with high charge states. The charge states in ICMEs also increase with solar activity levels. Other charge state variations (e.g., $\mathrm{C}^{5} / \mathrm{C}^{6}$ ) in some ICMEs are spatially related to variations in $T_{p} / T_{e x}$, suggesting that the $T_{p}$ signatures at 1 AU may occasionally contain vestiges of conditions during CME formation. An interesting subset of high Fe charge state events appear to form a corotating sequence, and further investigation of the associated solar wind structures is required.

\section{ACKNOWLEDGEMENTS}

We acknowledge the use of ACE MAG, SWEPAM and SWICS data from the ACE Science Center.

\section{REFERENCES}

1. Gosling, J. T., "Coronal mass ejections and magnetic flux ropes in interplanetary space," in Physics of Magnetic Flux Ropes, Geophys. Monogr. Ser., vol. 58, edited by C. T. Russell, E. R. Priest, and L. C. Lee, Washington, D.C., AGU, 1990, p. 343.

2. Richardson, I. G., and Cane, H. V., J. Geophys. Res., 100, 23,397 (1995).

3. Richardson, I. G., Dvornikov, V. M., Sdobnov, V. E., and Cane, H. V., J. Geophys. Res., 105, 12,597 (2000).

4. Zwickl, R. D., J. R. Asbridge, S. J. Bame, W. C. Feldman, J. T. Gosling, and E. J. Smith, "Plasma properties of driver gas following interplanetary shocks observed by ISEE 3", in Solar Wind Five, NASA Conf. Pub., CP-2280, 1983, p. 711.

5. Lepri, S. T., Zurbuchen, T. H., Fisk, L. A., Richardson, I. G., Cane, H. V., and Gloeckler, G., J. Geophys. Res., 106, 29,231 (2001).

6. Richardson, I. G., Dvornikov, V. M., Sdobnov, V. E., and Cane, H. V., Proc. $27^{\text {th }}$ Int. Cosmic Ray Conf., 9, 3498 (2001).

7. Gosling, J. T., Birn, J., and Hesse, M., Geophys. Res. Lett., 22, 869 (1995). 